\title{
Mineralogy of air-pollution-control residues from a secondary lead smelter: environmental implications
}

\section{SUPPORTING INFORMATION}

\author{
Vojtěch Ettler* ${ }^{1}$, Zdenek Johan ${ }^{2}$, Alain Baronnet ${ }^{3}$, Filip Jankovský ${ }^{1,4}$, \\ Christian Gilles ${ }^{2}$, Martin Mihaljevič ${ }^{1}$, Ondřej Šebek ${ }^{5}$, Ladislav Strnad ${ }^{5}$ \\ and Petr Bezdička ${ }^{6}$
}

1 Institute of Geochemistry, Mineralogy and Mineral Resources, Charles University, Albertov 6, 12843 Praha 2, Czech Republic (e-mail: ettler@natur.cuni.cz, fax +420 221951 496, telephone +42 0221951 493)

2 Bureau des Recherches Géologiques et Minières (BRGM), av. Claude Guillemin, 45060 Orléans, cedex 2, France

3 Centre de Recherche en Matière Condensée et Nanosciences (CRMCN-CNRS), Campus de Luminy, 13288 Marseille, cedex 09, France

4 Geotest Brno, Office of Prague, Máchova 23, 12000 Praha 2, Czech Republic

5 Laboratory of Geological Departments, Charles University, Albertov 6, 12843 Praha 2, Czech Republic

${ }^{6}$ Institute of Inorganic Chemistry, Academy of Science of the Czech Republic, 25068 Rež, Czech Republic

*corresponding author 


\section{Standards and detection limits of electron microprobe (EPMA):}

The following standards were used for silicates and oxides: albite ( $\mathrm{Si}, \mathrm{Na}), \mathrm{K}$-feldspar $(\mathrm{K})$, forsterite $(\mathrm{Mg})$, hematite $(\mathrm{Fe})$, andradite $(\mathrm{Ca}), \mathrm{ZnO}(\mathrm{Zn})$, vanadinite $(\mathrm{Pb}, \mathrm{Cl})$, synthetic $\mathrm{Cr}_{2} \mathrm{O}_{3}$ (Cr), barite (S), synthetic $\mathrm{MnTiO}_{3}(\mathrm{Ti}, \mathrm{Mn})$, synthetic $\mathrm{AsGa}(\mathrm{As}), \mathrm{Al}_{2} \mathrm{O}_{3}(\mathrm{Al})$, stibnite (Sb), cassiterite $(\mathrm{Sn})$, copper $(\mathrm{Cu})$ and cadmium $(\mathrm{Cd})$ metals. The detection limits for the individual elements are as follows (in wt.\%): $0.02(\mathrm{Si}), 0.03(\mathrm{Na}, \mathrm{Al}, \mathrm{Mg}), 0.04(\mathrm{~K}, \mathrm{Cl}), 0.06(\mathrm{Ca}), 0.07$ (Ti, S), 0.11 (As), 0.13 (Fe), $0.14(\mathrm{Sn}), 0.15$ (Cd, Cr), 0.18 (Mn), $0.20(\mathrm{Sb}), 0.26(\mathrm{~Pb}), 0.27$ $(\mathrm{Cu}), 0.39(\mathrm{Zn})$.

\section{Note concerning the interpretation of EPMA:}

EPMA revealed strong variations in the major element concentrations, suggesting that most of the analyzed points are composed of several phases, especially for extremely fine-grained materials (samples A3 and A4). For this reason, interpretation of the analytical data took into consideration the results of XRD (Table 2) in order to proceed to a model calculation of mol \% of the individual chemical compounds in a particular analysis (Table S1). Some of the analytical points yielded low totals due to the extremely fine-grained nature of the analyzed material, especially in the matrices of sample A4 (Table S3, analyses 10 to 13). 
Table S1. Microprobe Analyses of Pb-rich Glass, $\mathrm{Pb}$ Sulfates, Laurionite and Galena in Weight \% (wt.\%) and in Atomic \% (at.\%)

\begin{tabular}{|c|c|c|c|c|c|c|c|}
\hline analysis & $1^{a}$ & $2^{b}$ & $3^{b}$ & $4^{c}$ & $5^{d}$ & $6^{\mathrm{e}}$ & $7^{f}$ \\
\hline sample & A1 & A2 & A4 & $\mathrm{A} 2$ & A2 & A2 & A2 \\
\hline \multicolumn{8}{|l|}{ wt.\% } \\
\hline $\mathrm{Pb}$ & 10.57 & 69.34 & 68.51 & 49.46 & 69.79 & 82.11 & 87.86 \\
\hline$S$ & - & 10.69 & 10.56 & 12.61 & 9.76 & - & 11.25 \\
\hline O & 41.30 & 21.44 & 21.30 & 25.63 & 20.16 & 6.39 & - \\
\hline $\mathrm{Cl}$ & 0.16 & - & 0.27 & - & 0.23 & 15.36 & 0.19 \\
\hline $\mathrm{Cl} \equiv \mathrm{O}$ & -0.04 & - & -0.06 & - & -0.05 & -3.53 & -0.04 \\
\hline $\mathrm{Na}$ & 0.06 & - & - & 0.08 & 0.05 & - & - \\
\hline K & 0.42 & - & 0.06 & 10.22 & - & - & - \\
\hline $\mathrm{Ca}$ & 0.13 & - & 0.11 & 1.13 & - & - & - \\
\hline Si & 20.28 & 0.03 & - & 0.05 & - & - & 0.07 \\
\hline $\mathrm{Ti}$ & 0.35 & - & - & - & - & - & - \\
\hline Al & 18.66 & - & - & - & - & - & - \\
\hline $\mathrm{Fe}$ & 0.61 & - & - & - & - & - & - \\
\hline $\mathrm{Mg}$ & 0.15 & - & - & - & - & - & - \\
\hline Zn & - & - & - & 0.58 & - & - & 0.50 \\
\hline $\mathrm{Sb}$ & - & - & - & 0.24 & - & - & 0.32 \\
\hline Sn & - & - & - & 0.21 & - & - & - \\
\hline As & - & - & 0.26 & - & 0.22 & - & - \\
\hline Total & 92.65 & 101.51 & 101.01 & 100.22 & 100.15 & 100.32 & 100.14 \\
\hline \multicolumn{8}{|l|}{ at. $\%$} \\
\hline $\mathrm{Pb}$ & 1.25 & 16.66 & 16.48 & 9.39 & 17.61 & 32.25 & 53.49 \\
\hline S & - & 16.59 & 16.42 & 15.47 & 15.91 & - & 44.26 \\
\hline O & 63.09 & 66.69 & 66.33 & 63.05 & 65.87 & 32.48 & - \\
\hline $\mathrm{Cl}$ & 0.11 & - & 0.38 & - & 0.35 & 35.27 & 0.66 \\
\hline $\mathrm{Na}$ & 0.06 & - & - & 0.14 & 0.12 & - & - \\
\hline K & 0.26 & - & 0.08 & 10.29 & - & - & - \\
\hline $\mathrm{Ca}$ & 0.08 & - & 0.14 & 1.09 & - & - & - \\
\hline Si & 17.65 & 0.06 & - & 0.06 & - & - & 0.30 \\
\hline $\mathrm{Ti}$ & 0.18 & - & - & - & - & - & - \\
\hline Al & 16.91 & - & - & - & - & - & - \\
\hline $\mathrm{Fe}$ & 0.27 & - & - & - & - & - & - \\
\hline $\mathrm{Mg}$ & 0.15 & - & - & - & - & - & - \\
\hline Zn & - & - & - & 0.35 & - & - & 0.96 \\
\hline $\mathrm{Sb}$ & - & - & - & 0.08 & - & - & 0.33 \\
\hline Sn & - & - & - & 0.07 & - & - & - \\
\hline As & - & - & 0.17 & - & 0.15 & - & - \\
\hline \multicolumn{8}{|c|}{$\begin{array}{l}\text { a } \mathrm{Ca} \text {-Fe silicate glass with high concentration of } \mathrm{Pb} \\
\text { b anglesite } \mathrm{PbSO}_{4} \\
\text { c ideally correspons to the compound } \mathrm{K}_{2} \mathrm{SO}_{4} \cdot 2 \mathrm{PbSO}_{4} \\
\text { d ideally corresponds to } \mathrm{PbO} \cdot 1 \mathrm{OPSO} \mathrm{PbO}_{4} \\
\left.\text { e ideally corresponds to laurionite } \mathrm{Pb}_{4} \mathrm{OH}\right) \mathrm{Cl} \\
\mathrm{f} \text { galena (PbS) with excess of } \mathrm{Pb} \text { with respect to stoichiometric formula (probably submicrometric } \mathrm{Pb} \\
\text { exsolutions in the } \mathrm{Pb}-\mathrm{PbS} \text { system) } \\
\text { - not detected }\end{array}$} \\
\hline
\end{tabular}




\begin{tabular}{|c|c|c|c|c|}
\hline \multicolumn{5}{|c|}{$\begin{array}{l}\text { Table S2. Variations in Microprobe Analyses of } \\
\text { the Fine-grained Residue A3 in Weight \% (wt.\%) } \\
\text { and in Atomic \% (at.) }\end{array}$} \\
\hline wt.\% & mean & $S^{a}$ & $\min$ & $\max$ \\
\hline $\mathrm{Pb}$ & 41.91 & 4.91 & 34.20 & 49.76 \\
\hline$S$ & 2.06 & 0.47 & 1.32 & 2.93 \\
\hline O & 7.49 & 1.10 & 5.99 & 9.56 \\
\hline $\mathrm{Cl}$ & 11.86 & 2.54 & 5.31 & 16.99 \\
\hline $\mathrm{Cl} \equiv \mathrm{O}$ & -2.68 & -0.57 & -1.20 & -3.83 \\
\hline $\mathrm{Na}$ & 0.67 & 0.19 & 0.29 & 0.96 \\
\hline K & 1.88 & 0.89 & 0.95 & 4.93 \\
\hline $\mathrm{Ca}$ & 0.13 & 0.05 & - & 0.27 \\
\hline $\mathrm{Si}$ & 0.12 & 0.05 & 0.07 & 0.24 \\
\hline $\mathrm{Al}$ & 0.03 & 0.01 & - & 0.05 \\
\hline $\mathrm{Fe}$ & 0.27 & 0.08 & - & 0.42 \\
\hline $\mathrm{Zn}$ & 0.41 & 0.02 & - & 0.43 \\
\hline $\mathrm{Cd}$ & 0.69 & 0.23 & - & 0.83 \\
\hline $\mathrm{Sb}$ & 0.28 & 0.09 & - & 0.47 \\
\hline Sn & 0.46 & 0.21 & 0.25 & 0.97 \\
\hline As & 0.12 & 0.01 & - & 0.14 \\
\hline Total & 64.82 & 5.55 & 55.88 & 72.33 \\
\hline \multicolumn{5}{|l|}{ at.\% } \\
\hline $\mathrm{Pb}$ & 17.38 & 1.89 & 14.33 & 20.35 \\
\hline S & 5.48 & 0.93 & 3.34 & 7.11 \\
\hline O & 40.01 & 3.84 & 32.28 & 48.61 \\
\hline $\mathrm{Cl}$ & 28.82 & 6.21 & 12.18 & 39.06 \\
\hline $\mathrm{Na}$ & 2.52 & 0.73 & 1.04 & 3.41 \\
\hline $\mathrm{K}$ & 4.09 & 1.78 & 2.28 & 10.27 \\
\hline $\mathrm{Ca}$ & 0.27 & 0.11 & - & 0.54 \\
\hline $\mathrm{Si}$ & 0.37 & 0.15 & 0.19 & 0.76 \\
\hline $\mathrm{Al}$ & 0.11 & 0.02 & - & 0.14 \\
\hline $\mathrm{Fe}$ & 0.42 & 0.12 & - & 0.62 \\
\hline $\mathrm{Zn}$ & 0.49 & 0.03 & - & 0.54 \\
\hline $\mathrm{Cd}$ & 0.52 & 0.18 & - & 0.68 \\
\hline $\mathrm{Sb}$ & 0.19 & 0.06 & - & 0.31 \\
\hline Sn & 0.33 & 0.14 & 0.17 & 0.61 \\
\hline As & 0.13 & 0.02 & - & 0.15 \\
\hline
\end{tabular}


Table S3. Microprobe Analyses of Caracolite-Anglesite Mixture, Spinel, FeO and Complex Matrix Phases in Weight \% (wt.\%) and in Atomic \% (at.\%)

\begin{tabular}{lcccccc} 
analysis & $\mathbf{8}^{\mathbf{b}}$ & $\mathbf{9}^{\mathbf{c}}$ & $\mathbf{1 0}^{\mathbf{d}}$ & $\mathbf{1 1}^{\mathbf{e}}$ & $\mathbf{1 2}^{\mathbf{e}}$ & $\mathbf{1 3}^{\mathbf{e}}$ \\
sample & $\mathbf{A 4}$ & $\mathbf{A 4}$ & $\mathbf{A 4}$ & $\mathbf{A 4}$ & $\mathbf{A 4}$ & $\mathbf{A 4}$ \\
\hline wt.\% & & & & & & \\
$\mathrm{Pb}$ & 57.07 & 2.35 & 4.77 & 20.97 & 34.66 & 20.20 \\
$\mathrm{~S}$ & 11.09 & - & 0.08 & 0.08 & 0.17 & - \\
$\mathrm{O}$ & 22.96 & 19.79 & 18.85 & 14.79 & 13.51 & 14.03 \\
$\mathrm{Cl}$ & 2.13 & 0.14 & 0.81 & 3.20 & 1.29 & 1.22 \\
$\mathrm{Cl}=\mathrm{O}$ & -0.48 & -0.03 & -0.18 & -0.72 & -0.29 & -0.28 \\
$\mathrm{Na}$ & 2.58 & 1.00 & 0.29 & 0.83 & 0.59 & 0.78 \\
$\mathrm{~K}$ & 1.69 & - & 0.09 & 0.15 & 0.57 & 0.57 \\
$\mathrm{Ca}$ & 0.13 & - & - & - & - & - \\
$\mathrm{Si}$ & - & 0.05 & 0.09 & 0.06 & 0.07 & 0.07 \\
$\mathrm{Al}$ & - & - & 0.05 & 0.06 & 0.07 & 0.04 \\
$\mathrm{Fe}$ & - & - & 46.30 & 0.30 & 0.31 & 0.43 \\
$\mathrm{Mg}$ & - & 0.05 & - & 0.05 & 0.05 & - \\
$\mathrm{Cu}$ & - & - & 0.54 & 0.56 & - & - \\
$\mathrm{Zn}$ & - & 39.71 & 16.66 & 28.89 & 14.92 & 16.13 \\
$\mathrm{Cd}$ & - & - & - & 0.76 & 3.41 & 4.57 \\
$\mathrm{Sb}$ & - & 3.15 & 2.34 & 12.49 & 21.27 & 24.12 \\
$\mathrm{Sn}$ & - & 31.92 & - & 5.86 & 4.44 & 7.02 \\
$\mathrm{As}$ & 1.72 & 0.36 & 0.38 & 2.20 & 0.95 & 1.14 \\
$\mathrm{Total}$ & 98.90 & 98.49 & 91.07 & 90.53 & 95.99 & 90.04 \\
at.\% & & & & & & \\
$\mathrm{Pb}$ & 11.98 & 0.51 & 0.97 & 5.60 & 10.52 & 5.99 \\
$\mathrm{~S}$ & 15.06 & - & 0.10 & 0.14 & 0.33 & - \\
$\mathrm{O}$ & 62.44 & 56.06 & 49.85 & 51.10 & 53.12 & 53.84 \\
$\mathrm{Cl}$ & 2.61 & 0.18 & 0.96 & 4.99 & 2.29 & 2.11 \\
$\mathrm{Na}$ & 4.88 & 1.98 & 0.54 & 2.00 & 1.61 & 2.08 \\
$\mathrm{~K}$ & 1.88 & - & 0.10 & 0.21 & 0.92 & 0.90 \\
$\mathrm{Ca}$ & 0.14 & - & - & - & - & - \\
$\mathrm{Si}$ & - & 0.08 & 0.14 & 0.12 & 0.16 & 0.15 \\
$\mathrm{Al}$ & - & - & 0.08 & 0.12 & 0.16 & 0.09 \\
$\mathrm{Fe}$ & - & - & 35.08 & 0.30 & 0.35 & 0.47 \\
$\mathrm{Mg}$ & - & 0.09 & - & 0.11 & 0.13 & - \\
$\mathrm{Cu}$ & - & - & 0.36 & 0.49 & - & - \\
$\mathrm{Zn}$ & - & 27.52 & 10.79 & 24.43 & 14.36 & 15.15 \\
$\mathrm{Cd}$ & - & - & - & 0.37 & 1.91 & 2.50 \\
$\mathrm{Sb}$ & - & 1.17 & 0.81 & 5.67 & 10.99 & 12.16 \\
$\mathrm{Sn}$ & - & 12.19 & - & 2.73 & 2.35 & 3.63 \\
$\mathrm{As}$ & 1.00 & 0.22 & 0.22 & 1.62 & 0.80 & 0.93 \\
$\mathrm{H}$ & & & & & &
\end{tabular}

\section{mol.\%}

\begin{tabular}{|c|c|c|c|c|c|c|}
\hline caracolite & 12.2 & - & - & - & - & - \\
\hline$(\mathrm{K}, \mathrm{Na}) \mathrm{Cl}$ & - & 12.0 & 1.3 & 8.4 & 14.8 & 16.0 \\
\hline $\mathrm{PbCl}_{2}$ & 7.4 & - & 0.4 & 5.3 & - & - \\
\hline $\mathrm{PbSO}_{4}$ & 76.7 & - & 0.2 & 0.5 & 1.9 & - \\
\hline$(\mathrm{Sb}, \mathrm{As})_{2} \mathrm{O}_{3}$ & 3.7 & 4.2 & 1.1 & 13.9 & 34.4 & 35.2 \\
\hline spinel & - & 83.8 & 1.5 & 25.8 & 48.9 & 48.8 \\
\hline$(\mathrm{Fe}, \mathrm{Zn}) \mathrm{O}$ & - & - & 95.5 & 46.1 & - & - \\
\hline
\end{tabular}



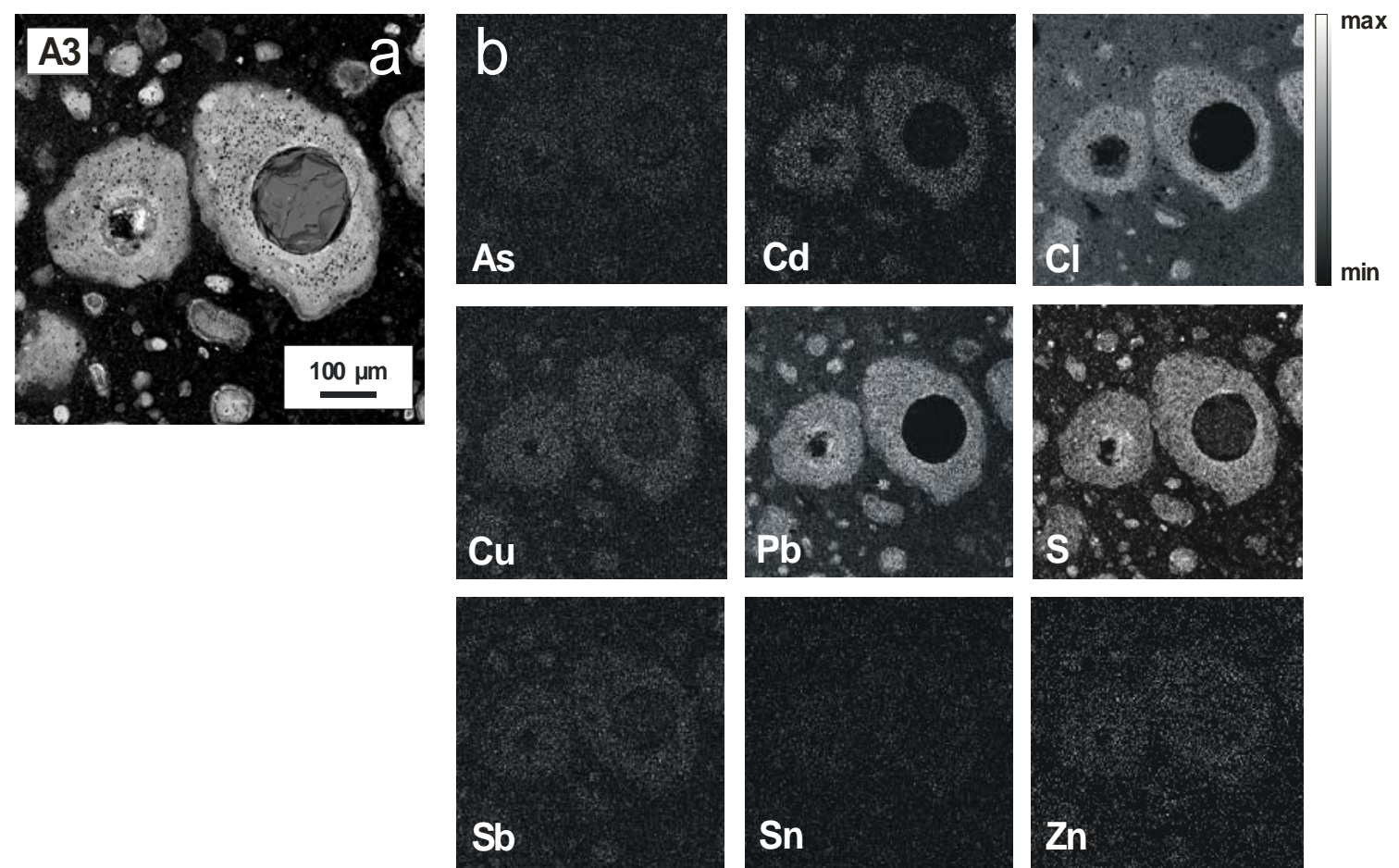

Figure S1. Sample A3 from bag filters. a) SEM image of accumulations of fine-grained $\mathrm{Pb}-$ rich material (potassium lead chloride and caracolite) with silicate glass inclusion (in backscattered electrons); b) X-ray maps showing the relative distribution of $\mathrm{As}, \mathrm{Cd}, \mathrm{Cl}, \mathrm{Cu}, \mathrm{Pb}, \mathrm{S}$, $\mathrm{Sb}, \mathrm{Sn}$ and $\mathrm{Zn}$.
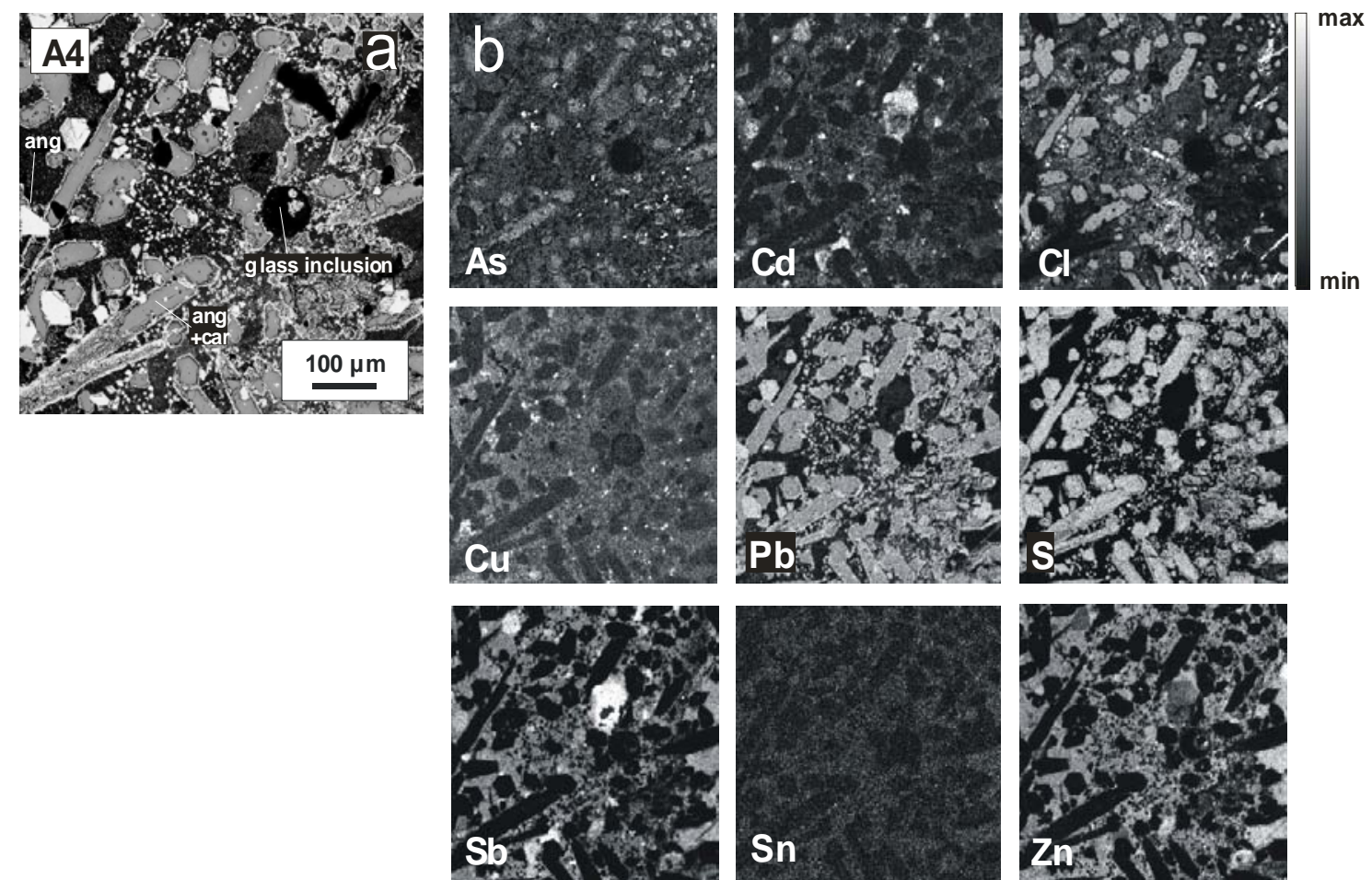

Figure S2. Sample A4 resulting from sintering of sample A3 at $\sim 500^{\circ}$ C. a) SEM image showing white anglesite (ang) crystals, needles of anglesite-caracolite (ang+car) mixture and residual silicate glass spheres within the dark gray matrix enriched in $\mathrm{Zn}, \mathrm{Sb}, \mathrm{Sn}$ and $\mathrm{Cd}$ (in back-scattered electrons); b) X-ray maps showing the relative distribution of $\mathrm{As}, \mathrm{Cd}, \mathrm{Cl}, \mathrm{Cu}$, $\mathrm{Pb}, \mathrm{S}, \mathrm{Sb}, \mathrm{Sn}$ and $\mathrm{Zn}$. 